\title{
EKMAN-HARTMANN BOUNDARY LAYERS AND THE LENGTH OF DAY VARIATIONS
}

\author{
N. KLEEORIN \\ Department of Mechanical Engineering, Ben-Gurion University \\ of Negev, POB 653, 84105 Beer-Sheva, Israel
}

I. ROGACHEVSKII

Racah Institute of Physics, Hebrew University of Jerusalem, 91904 Jerusalem, Israel

\section{A. RUZMAIKIN}

Isaac Newton Institute for Mathematical Sciences, Cambridge University, 20 Clarkson Road, CB2 0EH, Cambridge, U.K.

In the first issue of Journal of Fluid Mechanics Ian Proudman published a paper on the dynamical properties of a fluid between two concentric rotating spheres (Proudman, 1956). The angular velocities of the spheres were assumed only slightly different and the Reynolds number of the flow was large. It was found under non-slip boundary conditions that the cylindrical surface that touches the inner sphere and parallel to the axis of rotation is a singular surface in which velocity gradients are very large. Outside the cylinder the fluid rotates as a rigid body with the same angular velocity as the outer sphere. Inside the cylinder the fluid rotates with an angular velocity intermediate to the angular velocities of the spheres and there is also a meridional circulation. Later Stewartson (1966) presented a detailed investigation of structure of the shear layer near the cylindrical surface. One of the present authors (Ruzmaikin, 1989) pointed out a possible geophysical importance of these solutions. The liquid part of the Earth core occupying a shell between the inner solid core and the rock mantle can be considered as the fluid between two rotating spheres.

Actually, this fluid is well conducting and there is a magnetic field so the MHD approach is needed. It was first put forward by Bullard et al. (1950) that the dynamo generated magnetic field provides a coupling between the inner core and the mantle at a small difference between their angular velocities. This difference was identified with a rate of the observed westward drift of the Earth's magnetic field. Thus there is a good motivation to study MHD flows between two rotating spheres. 
In this paper we take into account all boundary and shear layers between two concentric spheres co-axially rotating with different angular velocities (see, the details in Kleeorin et al., 1992). The axisymmetric velocity and magnetic field in a conducting fluid are found. The boundaries of the spheres are assumed to have variable conductivities. There are three Ekman-Hartmann spherical boundary layers and one internal cylindrical shear layer. The magnetic fields in the Ekman-Hartmann layers cause an electromagnetic relaxation of differential rotation of the fluid while magnetic fields in the fluid outside the boundary layers can excite the differential rotation. This extends the results on electromagnetic coupling obtained in the geophysical context (Loper,1975; Braginsky and Fishman, 1976; Stix and Roberts, 1984).

A new kind of the electromagnetic coupling due to a modification of the viscous force by the magnetic field is pointed out. The coupling is important for the excitation of the differential rotation in the fluid. The properties of the internal cylindrical shear layer are essentially modified by the magnetic field generated inside this layer.

The problem of the low frequency variations in the rotation of the Earth is considered (see, for example, Stacey, 1969, Braginsky, 1970, Lambeck, 1980, Rochester ,1984). It was found that the relative variations in the Earth's angular velocity are

$$
\frac{\delta \Omega_{m}}{\Omega} \simeq \frac{\tau B_{p} B_{T} R^{3}}{\Omega I}(1+\delta) \simeq(5 \div 10) \cdot 10^{-9},
$$

where $B_{p}$ and $B_{T}$ are the poloidal and toroidal components of the magnetic field, $\Omega$ and $R$ are the angular velocity and radius of the inner core, $I$ is the moment of inertia of the Earth. The relaxation time $\tau$ and parameter $\delta$ depend on the mechanism of the dissipation of the flow. The relaxation of the flow due to viscosity $\nu$ occuars for the time $\tau_{\nu} \simeq(\Omega \sqrt{E})^{-1}$, while the electromagnetic relaxation takes place for the time $\tau_{m} \simeq 8 \pi \rho \eta / B_{p}^{2}$. Here $E=\eta / \Omega R^{2}$ is the Ekman number, $\rho$ is the density of the fluid, $\eta$ is the magnetic diffusivity due to electrical conductivity of the fluid.

Thus, observed low frequency variations ( $<100$ years) in angular velocity of the mantle can be produced by the internal magnetic forces. It may be shown also that for the typical parameters of the 
Earth's liquid core the gravitational forces in the time intervals under consideration ( $<100$ years) could not contribute to the length of day variations. Note, the total angular momentum of the Earth practically does not change in the time intervals, however there is a redistribution of the angular momentum between the mantle, the fluid and the solid core. The magnetic and viscous forces play the main role in these processes.

\section{REFERENCES}

Braginsky, S.I. and Fishman, V.M. 1976 Geomagn. and Aeron. 16, 443.

Bullard, E.C., Freedman, C., Gellman, H. and Nixon, J. 1950 Phil. Trans. Roy. Soc. London A 243, 67.

Kleeorin, N., Rogachevskii, I. and Ruzmaikin, A. 1992 J. Fluid Mech. submitt.

Lambeck, K. 1980 The Earth's variable rotation. Cambridge University Press, Cambridge.

Loper, D.E. 1975 Phys. Earth and Planet. Inter. 11, 43.

Proudman, I. 1956 J. Fluid Mech. 1, 505.

Rochester, M.G. 1984 Phil. Trans. R. Soc. Lond. A 313, 95.

Ruzmaikin, A. 1989 Geomagn. and Aeron. 29, 299.

Stewartson, K. 1966 J. Fluid Mech. 26, 131.

Stix, M. and Roberts, P.H. 1984 Phys. Earth and Planet. Inter. 36, 49. 